\title{
Die Chaostage 1995 als politisches Spektakel \\ Medialität und Materialität urbaner riots
}

\author{
Yannick Kalff \\ Katharina Warda
}

Vor 20 Jahren erschütterten die Chaostage die Messestadt Hannover. In den bis dato schwersten Auseinandersetzungen der bundesdeutschen Nachtkriegsgeschichte, brachte eine subkulturelle Strömung aus Punks, Skinheads und anderen, die Ordnungsvorstellung der bürgerlichen Gesellschaft ins Wanken.

Die Chaostage heute noch einmal als riot zu betrachten, öffnet den Blick auf ihre materielle und mediale Wirkung. Dabei wird deutlich, wie eine Unruhe Sinnzusammenbrüche der lebensweltlich erfahrbaren sozialen Ordnung verursacht. Diese Sinnentleerung motivierte infolge der Chaostage 1995 eine mediale Mobilmachung, um neuen Sinn zuzuschreiben und zu verstehen, was auf der Straße faktisch passiert war. Der riot wurde dabei mit seiner eigenen Spektakularität konfrontiert, er wurde fremddefiniert und somit den Beteiligten eine eigene Darlegung ihres Handelns verwehrt. Die Punks griffen diesen Punkt auf und hielten der medialen Hysterie ihr karnevaleskes Zerrbild entgegen.

Ersteinreichung: 15. Juni 2015; Veröffentlichung online: 1. Mai 2016

An english abstract can be found at the end of the document.

In uns ein Feuer, noch ein Abenteuer, heute soll's sein.

Die Bullen dagegen, doch gerade deswegen mu $\beta$ es jetzt sein.

Mit uns im Abteil nur Abschaum dabei: Musik, Gejohle und Geschrei.

Wohin's uns gefällt, wir brauchen kein Geld.

Heute Hannover und morgen die Welt!

Terrorgruppe 1996: Wochenendticket.

Album: Melodien für Milliarden

Mit den vorangestellten Zeilen nimmt die Band Terrorgruppe Bezug auf eines der identitätsstiftendsten Ereignisse der Punkszene in Deutschland: die Chaostage 1995. Im Liedtext bezeichnen sich die Punks selbst als Abschaum. Gerade weil die Polizei gegen sie und ihre Ruhestörungen vorgeht, fahren sie nach Hannover und stürzen sich ins Abenteuer.

Anhand dieser Narration lassen sich bereits wesentliche Elemente des Konflikts und Ursachen der Chaostage benennen. Selbst- und Fremdbild der Punks schreiben sich hier als das ,Anormale‘, das ,Andere‘ der bürgerlichen Gesellschaft ein. Zusätzlich wird ein Verlangen nach Abenteuer erkennbar, welches die Ereignisse begleitete: „Es gibt eine Sehnsucht nach dem 
Ereignis. Es wird geschehen, geschah einst. Es wird alles anders sein, alles ist schon anders geworden. [...] eine seltsame, vergessene Intensität wird die Handelnden erfassen“ (Trawny 2011: 7). Dieses nostalgische Drängen auf ein ,Ereignis“ richtet sich gegen die eingefahrenen, bleiernen Verhältnisse am scheinbaren „Ende der Geschichte“ (Fukuyama 1992). Die Chaostage sind und waren keine Revolution(en), dennoch brechen sie mit einer monotonen und deterministischen Geschichtsschreibung, um selbst Geschichte zu ,machen'. Sie markieren einen riot, der mithilfe medialer Inszenierungen über die Ereignisse auf der Straße hinausreichte und zum Spektakel wurde.

Wir gehen in unserem Text der These nach, dass es bei diesem riot um mehr ging als um einen ungerichteten, sinnlosen Ausbruch von Gewalt, dessen vielleicht griffigste Forderung „Hannover zur Wiese machen“ (Spiritus Rector 1996b) darstellte. Es handelte sich aus unserer Sicht eher um einen Deutungskampf, in welchem die hegemoniale bürgerliche Position in ihrer Selbstdarstellung irritiert und somit die Grundkonstitution von Öffentlichkeit in Frage gestellt wurde.

Vor diesem Hintergrund gelingt es - angesichts aktueller Debatten über „linke Gewalt“ in urbanen Räumen -, eine weitere Deutungsebene freizulegen, welche Gewalt auf der Straße mit einer ihr vorgängigen sowie folgenden gesellschaftlich-strukturellen Gewalt ins Verhältnis setzt. Die Chaostage 1995 werden, begreift mensch sie als Spektakel, zu einem interessanten und bisher nur wenig betrachteten Exempel für bis heute andauernde Kämpfe um gesellschaftliche Bedeutungszusammenhänge, deren Wirksphäre weit über ihre urbanen Schauplätze hinausgeht. Sie ereignen sich in verschiedenen gesellschaftlichen Arenen, zu denen materiell-symbolische Wirklichkeiten, wie die Straße, ebenso zählen wie mediale Öffentlichkeiten. Für die Ausdehnung von riots auf mediale Räume stellen die Chaostage 1995 zudem ein frühes und vielschichtiges Beispiel dar.

Zunächst werden wir die Begriffe riot, Gewalt und Spektakel in enger Relation zueinander definieren, um einen Deutungsrahmen bereitzustellen (1). Ein kurzer historischer Abriss der Punkkultur und der Chaostage vergegenwärtigt sodann das über zwanzig Jahre zurückliegende Geschehen (2). In den Abschnitten (3) und (4) untersuchen wir die Chaostage mit Fokus auf ihre symbolisch-materielle Beziehung zur Straße und ihre Medienstrategie und rekonstruieren sie als politisches Spektakel. Hierbei unterscheiden wir zwischen systemstabilisierenden und subversiven Inszenierungen. Beide lassen sich gleichermaßen finden und bestimmen hinsichtlich ihrer Wirkungsweise zentrale Kriterien eines riots.

\section{Riots, Gewalt, Spektakel: ein Setting}

Die Chaostage sind ein riot, also eine Ausschreitung, die in einem urbanen Raum stattfindet. Sie sind gewalttätig in dem Sinne, dass unterschiedliche Gruppen auf verschiedenen Ebenen verschiedene Formen von Gewalt mehr oder weniger zielgerichtet, anwenden'. Sie sind außerdem ein Spektakel, das eine spezifische Form von Happening suggeriert, bei welchem ,Unruhe‘ und ,Gewalt` zusammentreffen und eine Irritation produzieren. Im Folgenden erläutern wir zunächst die Begriffe riot, Gewalt und Spektakel und setzen sie dann zueinander in Beziehung. 
Von einer allgemeinen ,riots-Theorie‘ kann nicht die Rede sein. Vielmehr spielen sozio-strukturelle, historische sowie politische Verhältnisse eine Rolle, sodass die Begriffsbedeutung entsprechend dem jeweiligen Erkenntnisinteresse konzeptualisiert werden muss. Steffen Liebig (2014) beispielsweise stellt riots - in einer Untersuchung der englischen riots von 2011 - aus klassenspezifischer Sicht als Wandel von sozialen Konflikten dar. Von Liebig, aber auch von anderen Autor_innen, wird ferner nach dem Ursprung von riots gefragt. Demgegenüber fragen wir, wie sie funktionieren und welchen gesellschaftlichen sowie lokalen Verhandlungsmechanismen sie unterliegen, ohne dass wir eine Pathologisierung funktionalistischer Strukturen vornehmen (Pettenkofer 2010: 9).

Unruhen werden durch die Sprecher_innenpositionen inhaltlich gefüllt. Wie Iris Dzudzek und Michael Müller in ihrer Analyse der Londoner riots von 2011 feststellen, lüden unterschiedliche Positionen Unruhen verschieden auf (2013: 18). Die Zuschreibungen von Zielen, Motiven und politischen Positionen vollziehe sich in einem Spannungsfeld. Sie fänden zudem nicht unmittelbar in der Situation statt, sondern mittelbar und in kurzen Abständen zu den Ereignissen. Riots zu untersuchen bedeute daher zuallererst, ,,auf Spurensuche nach Selbstäußerungen und Brüchen im hegemonialen Repräsentationsregime der riots zu gehen“ (2013: 19).

Ein riot ist nach unserem Verständnis ein Deutungs-,moment', in welchem sich konfligierende Interpretationen begegnen und um Deutungs,hoheit' darüber gerungen wird, wie das - durch den Zusammenbruch sozialer Ordnung entstehende - Vakuum zu füllen ist. Da dies nicht direkt in der Situation des Bruchs geschieht, ist der eigentliche riot zunächst ,unbeschrieben'; er ist ,sinnlos', da er keine anschlussfähige Botschaft transportiert. Er widersetzt sich somit den bürgerlich-politischen Verfahrensweisen und Praktiken, demokratisch legitimen Widerspruch zu üben.[1] Dieser Umstand ist ein Problem: Als faktisches Ereignis kann der riot nicht unkommentiert bleiben, das heißt ohne Sinn. Riots müssen definiert werden. Das Sinnlos-Ambivalente nicht aushalten zu können, konstituiere laut Bauman die bürgerlich-moderne Gesellschaft wiederum grundlegend (1992), was sich im steten Drang, das ,Unfassbare‘ zu benennen und zu kategorisieren, niederschlage. Erst wenn Sinn zugeschrieben worden ist, kann der Unruhe mit Maßnahmen begegnet werden. Das Ereignis wird also retrospektiv mit einer Narration verbunden, um den Zusammenbruch der lebensweltlichen Ordnungserfahrung zu kitten.

Ferdinand Sutterlüty (2013) bezieht sich im Kontext der riots in Frankreich von 2005 und denen in England von 2011 auf Gary Marx und nutzt den Terminus issueless riots; er spricht im Falle Frankreichs auch vom „Nihilismus der Gewalt“ (2013: 10). Auf Slavoj Žižek (2005) verweisend, hält Sutterlüty fest, dass ein konkreter Sinn in den Geschehnissen selbst nicht zu suchen sei. David Harvey bezeichnet die englischen rioter als „,[n]ihilistic and feral teenagers“ (2012: 155). Darüber hinaus interpretiert er das Verhalten der Jugendlichen als Nachahmung der ihnen widerfahrenden kapitalistischgesellschaftlichen Gewalt, die sie so direkter erfahrbar und sichtbar machen würden (2012: 156f.). Allgemein gesprochen ist ein riot also ein Ausbruch 
von Gewalt, dem oftmals wiederum mit Gewalt begegnet wird. Gegenüber einer Revolution, einer Revolte oder einer Demonstration haben riots kein konkretes Ziel. Sie sind nicht Mittel zum Zweck, sie sind Selbstzweck.

\section{Gewalt}

Gewalt ist ein facettenreicher Begriff. Um ihn in Verbindung mit riots zu denken, zeichnen wir daher zunächst einige Konturen der Debatte über ihn nach.

(1) In sozialwissenschaftlichen Theorien wird der Gewaltbegriff kaum einzeln thematisiert. Allerdings sei er „Lieblings[kind] der Soziologie der Abweichung, der Kriminalität und der sozialen Probleme“ (Trotha 1997: 16). Mainstream und kritische Forschung stellen für Birgitta Nedelmann (1997: 60) dabei zwei Pole dar, zwischen denen Gewalt als (Teil-)Gegenstand verschiedener (Teil-)Disziplinen verhandelt werde; einerseits im Kontext „formalpolitische[r] Entscheidungsstrukturen“, andererseits im Zusammenhang mit einer „Gewaltforschung als eigenständiges sozialwissenschaftliches Gebiet“ (1997: 60). Diese Teilung begründe sich in der jeweiligen Interpretation von Gewalt: Wo sie als Element auftrete, welchem ,begegnet' werden muss, sieht Nedelmann einen Mainstream am Werk, welcher sie als notwendigen Aspekt sozialer Ordnung ausblende. Diese könne ohne Gewalt allerdings nicht hergestellt beziehungsweise aufrechterhalten werden (1997: 64f.). Gewalt ist demzufolge ein zentraler, dennoch latent bleibender, struktureller Faktor, der vom „Verschwindenlassen“ (Weller 2003: 500) bedroht ist.

(2) In der Moderne scheint Gewalt anachronistisch zu sein und doch stellt sie sich als das „Problem der Soziologie“ (Reemtsma 2006: 1) überhaupt dar, das nach der Konstitution von moderner Gesellschaft und ihrer Ordnung fragt. Gewaltforschung läuft, von der Annahme Gewalt sei anachronistisch ausgehend, Gefahr, spezifische gesellschaftliche Ordnungsvorstellungen und Selbstbeschreibungen zu reifizieren, die als explizit aufklärerischnormative Grundannahmen gesetzt werden. Gewalt wird somit einseitig als Scheitern dieser Ordnung gedeutet. Die Relation von Ordnung und Gewalt ist jedoch konstitutiv für die Moderne, denn Ordnung - als das Ausschließen von Ambivalenz (Bauman 1992) - erfordert gewaltvolle Interventionen (Sofsky 1996: 19ff.). Dieser Prozess führt zu einem ,infiniten Progreß der Gewalt“ (1996: 21). Ähnlich findet sich dies auch bei Žižek (2011), der „subjektive“ und „objektive“ Gewalt unterscheidet. Mit dieser Unterteilung beschreibt er die „Störung der ,normalen', friedvollen Ordnung der Dinge“ auf der einen und die „Nullebene“ (2011: 10) - vor welcher Handlungen als von der Norm abweichend, also als gewalttätig interpretiert werden können - auf der anderen Seite.

(3) Pettenkofer und Liell problematisieren für eine kulturwissenschaftliche Interpretation die potenzielle Ästhetisierung von Gewalt. Dieser ästhetisierende Blick, der sich von bisherigen Diskussionen über die Körperlichkeit von Gewalt und der Opferperspektive abwende, stoße sich immer noch an einer „unhintergehbaren Materialität“ (2004: 9). Als normative Übertretung ist Gewalt aber zuweilen ritueller Natur und nimmt die Form von „karnevalistischen Veranstaltungen“ (Dubet 1997: 221) an. Der Unterschied zu nicht-rituellen Formen liegt in der relativen Duldung dieser Abweichungen, insofern sie als Integrations- und Sozialisationsmechanismen nützen (auch 
Inhetveen 1997). Zudem folgt Gewalt, bei anthropologischer Betrachtung, kulturellen Regeln (Gabbert 2004: 88). Dies betont die „kulturelle Relativität“ von Gewaltkonzepten sowie die „Multiperspektivität von Gewalthandlungen“ (Gabbert 2004: 90).

Fruchtbar für die Interpretation der Chaostage erscheint uns, Gewalt aus einer relationalen Perspektive zu interpretieren. So argumentiert Sofsky, dass Gewalt ihr ,Ideal‘ übersteige: „Sie nimmt auch die Dinge ins Visier [...], die symbolische und materielle Kultur." (1996: 192) In gewalttätiger Zerstörung liege der Wunsch, die „Ordnung der Dinge“ umzustürzen mit dem Ziel „Chaos anzurichten“, was schlicht bedeute, „Unterschiede einzuebnen, Differenzen auszutilgen, die Abstände zwischen den Dingen zu eliminieren, damit ein wildes Durcheinander entsteht, ein Tumult der Stoffe, Kräfte und Zeichen" (1996: 193). Auch der riot entfaltet sich eruptiv in gewalttätigen Auseinandersetzungen gegen hegemoniale Deutungen und Symbole. In einer an Foucault (1974) orientierten Perspektive wird die (Re-)Produktion der gesellschaftlichen Macht- und Wissensformation durch Gewalt gestört. Die Stabilität der Ordnung wird kontingent.

\section{Spektakel}

Auch dem Begriff des Spektakels wird in der Theorie ambivalent begegnet. Trotz der vielfältigen Verwendung des Begriffs, besteht der Großteil der Literatur dazu in Kritik an gesellschaftlichen und politischen Spektakeln als Affektökonomien. Diese Kritiken gehen mit einer negativen Konnotation des Begriffs einher, durch die er mit einer selbstzweckhaften und direkten Affektproduktion gleichgesetzt und als einfache Unterhaltung abgetan wird. Die kritische Lesart wird außerdem von der Überzeugung getragen, dass sich spätkapitalistische Gesellschaften durch die „Spektakelisierung“ des Normalen genauso auszeichnet wie durch die Normalität des Spektakels (Bartz/Krause 2007). Gleichzeitig wird der Kapitalismus hier insgesamt weniger als System oder Glaube, denn als Spektakel an sich verstanden (Metz/ Seeßlen 2012).

In Die Gesellschaft des Spektakels entwickelte Guy Debord ein Bild der spektakulären Totalität: „Das ganze Leben der Gesellschaften, in welchen die modernen Produktionsbedingungen herrschen, erscheint als eine ungeheure Sammlung von Spektakel. Alles, was unmittelbar erlebt wurde, ist in eine Vorstellung gewichen“ (1996: 14). Dabei entwirft er eine Gesellschaft, die im Spektakel völlig aufgeht. Das Spektakel sei nämlich keine „abgesonderte Pseudowelt“, vielmehr „stellt [es] sich zugleich als die Gesellschaft selbst, als Teil der Gesellschaft und als Vereinigungsinstrument dar.“ (1996:14) Es sei „,nicht ein Ganzes von Bildern, sondern ein durch Bilder vermitteltes gesellschaftliches Verhältnis zwischen Personen“ (1996: 14). Bartz und Krause weisen in diesem Zusammenhang darauf hin, dass Debord hier - ähnlich wie Foucault - einer Idee von Macht folge, bei der diese weniger von außen auf Subjekte einwirke, als vielmehr internalisiert werde. Im Zuge dessen würden die Subjekte isoliert und die politische Kraft ihrer Körper reduziert, was schlussendlich Normalisierungseffekte zeitige (2007: 17). Trotz Debords theoretischer Unschärfe machen Bartz und Krause also von ihm ausgehend die Inszenierung von Normalität im Spektakel sichtbar. Eine besondere 
Rolle komme dabei der „Präsentation des Anormalen und Pathologischen“ (ebd: 19) zu. Das Spektakel des Anormalen und Pathologischen erscheine als ,wahre' Abbildung der Realität, wodurch aber wiederum erst festgelegt werde, was ,normal' ist und was nicht. Gleichzeitig werde das Dargestellte mit einem unerreichbaren Ideal von Normalität normativ aufgeladen.

„Da Normalisierungsverfahren aber keinen Zweck außerhalb ihrer selbst verfolgen, da sie nirgendwohin führen, können sie auch keine Energie aus der Formulierung von Zielvorstellungen gewinnen, sondern müssen sich aus eigener Kraft in Gang halten. Dies kann Normalisierung nur erreichen, indem sie [...] einerseits Normalität als unerreichbar darstellt, sie unaufhörlich neu erfindet, sich so dem auf sie zueilenden Individuum beständig entzieht und es dazu zwingt, sich als Individuum immer wieder neu zu bestätigen, und indem sie andererseits die Gefahr, nicht normal zu sein, regelmäßig aktualisiert, indem sie das Anormale in allen seinen Gestalten unablässig in Spektakeln vorführt.“ (Bartz/Krause 2007: 19)

An dieser Stelle setzt unser Verständnis von Spektakel an: Wir verstehen es als affekterzeugende Inszenierung und damit als zelebrierte Fiktion, deren Lustprinzip zwar Selbstzweck, aber auch nie frei von Wissens- und Sinnproduktion ist.

„Die Lust an der Fiktion und das Wissen, dass es sich um Fiktion handelt, gehören [...] zusammen. Das affektive und intellektuelle Moment schließen einander nicht aus. Vielmehr setzt das eine das andere voraus. Keine Lust ohne besseres Wissen. [...] Nur und gerade dann, wenn wir die Illusion des Spiels durchschauen, werden wir von dieser Illusion gepackt." (Pfaller 2002: 115f.)

Abgesehen vom kapitalisierenden und normalisierenden Nutzen, der aus einem Spektakel gezogen werden kann, ist auch eine Art Gegenspektakel möglich, das sich durch Spiegelung und Inversion gegen jede Vereinnahmung wehrt. In der Forschung wird für solche Inszenierungen der Begriff des Karnevalesken verwendet. Dabei wird der Karneval nicht als historischkulturelles Phänomen verstanden, sondern als ein Modus der lust- und humorvollen, spielerischen Verkehrung (Gau/Schlieben 2008).

„Der Karneval ist bestrebt, die Hegemonie von Ideologien, die das letzte Wort über die Welt haben wollen, aufzudecken, zu untergraben - ja zu zerstören - sowie das Leben zu erneuern, es samt der ihm innewohnenden Bedeutungen zu erhellen, Möglichkeiten aufzuzeigen. Er entwirft ein alternatives Konzept von Wirklichkeit.“ (Cuevas 2008: 290)

Das karnevaleske Spektakel entlarvt - mit den gleichen Unterhaltungstechniken wie sein normalisierender Konterpart - die Normalität konstruierende Sinngenese als ideologischen und damit vermachteten Prozess. Gleichzeitig macht es das Spektakel selbst als Affektökonomie sichtbar. Das Spektakel ist also ambivalent: Einerseits erhält es durch kapitalistische Verwertungs- und gesellschaftliche Normalisierungsinteressen eine vereinnahmende Funktion, andererseits ist es eine widerständige Praxis (Gau/Schlieben 2008). 


\section{Synthese}

Um die Chaostage zu interpretieren, nehmen wir die drei diskutierten Elemente (riot, Gewalt, Spektatel) als Ausgangspunkte und machen ihre Relationen sichtbar. So lässt sich zunächst Folgendes feststellen: Erstens wurden die Chaostage in verschiedenen Kontexten gedeutet: während der Ausschreitungen auf der Straße und in der Öffentlichkeit sowie in öffentlichen Diskussionen. Zweitens erschließt sich die Rolle von Gewalt auch hier nicht durch einseitige Betrachtungen von Täter_innen und/oder Opfern. Durch sie werden auch vorherrschende Kategorien, Klassifikationen und Differenzen angegriffen. Drittens entfaltete sich im antagonistischen Miteinander von bürgerlicher Gesellschaft und Punks die impulsive Dramaturgie von Spektakeln.

Vor allem die (Sach-)Beschädigungen im öffentlichen Raum wurden bei den Chaostagen von Punks und Medien in Szene gesetzt, um den riot als selbstversicherndes Spektakel nutzbar zu machen und eine öffentliche Ablehnung hervorzurufen. Was auf der Straße passierte, konnte und durfte nur im Einklang mit dem gesellschaftlichen Selbstverständnis als Symbol mit Sinn aufgeladen werden. Das heißt für die Chaostage, dass sie in der Öffentlichkeit als stupide, ziellose Unruhe definiert wurden, deren Protagonist_innen als reaktant psycho-pathologisiert wurden. Die subversive Strategie der Punks bestand darin, die bürgerlichen Wertvorstellungen zu (zer-)stören, indem sie sich der Sinngebung zunächst verwehrten und in einem weiteren Schritt neuen Sinn stifteten. Was die Chaostage somit von anderen riots unterschied, war ihre souveräne Fortführung. Die Teilnehmer_innen der Chaostage ,antworteten' auf die mediale Berichterstattung mit einem karnevalesken Gegenspektakel und erzwangen eine Auseinandersetzung auf anderen Ebenen, indem sie hegemoniale Zeichen der bürgerlichen Gesellschaft angriffen.[2]

\section{Punk und Chaos: die Chaostage in Hannover}

Punk entstand in den 1970er-Jahren und trat nach einer gewissen Latenzzeit ins gesellschaftliche Bewusstsein. In den 1980er und 1990er-Jahren wurde er auch zum Gegenstand (sozial-)wissenschaftlicher Diskussionen. Seit den 1990-er Jahren verliert er sich zwischen alternativen subkulturellen Angeboten. Insbesondere rechte Jugendgewalt dominiert nun das Forschungsinteresse (Eisenberg/Gronemeyer 1993). In aktuellen Studien, wie beispielsweise in einer Infoschrift der Landeszentrale für politische Bildung Thüringen, wird Punk als Jugendkultur zwischen „Kommerz \& Politik“ (Farin 1997) gesehen. Thomas Lau diskutiert zudem „Strukturmerkmale“ (1992: 119f.) des Punks und seiner Vorläufer, zu denen er Skinheads, Dadaismus, Narren und Franziskaner-Mönche zählt (1992: 124ff.). Aktuell bietet der Sammelband Punk in Deutschland sozial- und kulturwissenschaftliche Perspektiven auf Punk (Meinert/Seeliger 2013).

Die Untersuchungen zu Subkulturen werden insgesamt von jugendsoziologischen und pädagogischen Ansätzen dominiert, die unter anderem ihre Gewaltaffinität (Farin/Seidel 2012), Delinquenzen, Protestformen (Baacke 1985) oder ihre gesellschaftliche Stellung fokussieren (Hafeneger/ Stüwe/Weigel 1992, Köster 2014, Reimitz 1989, Schwell 2005). Ferner kommen 
deskriptive Studien hinzu, die Intention und Wertekanon von Subkulturen beleuchten sowie die Rituale beschreiben, wenn Punks ,auf die Spitzendeckchen der Nation“ (Soeffner 1992: 97) treten. Schriften der Landeszentrale(n) für politische Bildung beschäftigten sich seit dem Ende der 200oer-Jahre unter zeitgeschichtlichen Fragen mit der Punkszene (Hahn 2009; 2013, Westhusen 2005). Ein Lexikon macht Punk als Subkultur und seine Stellung als kulturelle Avantgarde greifbar (Graf 2003) und fragt nach zukünftigen Entwicklungen (Marcus 1992). Mittelbar wird sich auch in anderen Zusammenhängen auf Punk oder Punkrock bezogen, wobei beispielsweise intersektionale Themen fokussiert werden (Malott/Peña 2004, Sabin 1999).

Die Literatur bietet jedoch keinen systematischen Einblick in szenetypische ,Events' wie die Chaostage. Das zitierte Nachschlagewerk berichtet immerhin in einem Eintrag von den ersten Treffen ab 1982, die als „PunkerMesse“ in Hannover angelegt gewesen seien, um dieses „Lebensgefühl öffentlich zu vermitteln" (Graf 2003: 130). Dabei sei es bereits 1984 zu kurzen Ausschreitungen gekommen. Von 1985 bis 1993 pausierten die Chaostage und in den 1990er-Jahren hätten sie sich als „Gegenveranstaltung zur Berliner ,Love Parade““ (ebd.: 130) etablieren wollen.

Als ,Event' wurde die Veranstaltung in der Szene selbst bald ein Mythos, was sich auch auf ihre konstante Ausrichtung in Hannover zurückführen lässt (Geiling 1995: 1ff.). Sie sind dabei vor allem eine Reminiszenz für ältere Punk-Generationen (Bennett 2006: 220). Herbertz geht der Frage nach, wie die Chaostage ,organisiert' wurden, das heißt, wie sie als urbanes Happening Kontinuität erfahren haben. Dabei zeigt er die mediale Mobilisierung durch Flyer, Kettenbriefe, erste Internetpräsenzen sowie selbsterstellte FanZeitungen. Er skizziert Eckpunkte der diskursiven Verhandlungen der Chaostage sowie eine „Geschichte der Chaostage“ (Geiling 2000: 166ff., Herbertz 2011: 246ff., Meinert/Seeliger 2013: 39f.). Geiling rekonstruiert darüber hinaus aus der Berichterstattung lokaler und überregionaler Zeitungen eine „Punk-Phobie“ (2000: 173), welche „zum Marsch gegen die Feinde der Zivilisation“ (ebd.: 172) aufgerührt habe. Als letzter Ausweg habe deshalb nur noch der „Ausnahmezustand als Bewältigungsstrategie“ (ebd.: 175) geholfen, nachdem polizeiliche Deeskalationskonzepte 1995 gescheitert wären. Nicht zuletzt in diesen verschärften und hysterischen Reaktionen auf die Chaostage sieht Geiling ihre Mystifizierung begründet (1995: 6). Aus einer rechtswissenschaftlichen Perspektive werden wiederum die Verschärfung des Versammlungsrechts oder die vereinfachte Verhängung von Aufenthaltsverboten als Folge der Chaostage diskutiert (Seifert 1996). Unsere Analysen beziehen sich unter anderem deshalb auf die Vorkommnisse im Jahr 1995, weil sie die größte Debatte in den Medien nach sich gezogen haben.

\section{Materialität und Metaphorik der Straße und deren mediale Rezeption}

Als sich am ersten Augustwochenende 1995 Punks, Polizist_innen und Bundesbeamt_innen sowie Journalist_innen in der Messestadt Hannover einfanden, steuerte keiner einen festgelegten Ort an. Die Pressevertreter_innen suchten konfrontative Räume, Action und Sensation. Die Polizei versuchte, 
immer dort zu sein, wo die Punks waren. Diese wiederum hatten keinen vereinbarten Treffpunkt. Den Flyern ließen sich nur vage Informationen entnehmen: „,S]tatt einer klassischen Uhrzeitangabe wurde ,High Noon“ genannt und auch die Notwendigkeit, Treffpunkte anzugeben, [wurde] explizit verneint: ,Wo? Das weiß doch jeder!!!!!““ (Herbertz 2011: 252). Der Treffpunkt war wesentlich abstrakter und zugleich identitätsstiftender: die Straße. Hierbei handelte es sich nicht um eine bestimmte oder vorab festgelegte Straße oder einen bestimmten Raum, der einzelne Punkte der Stadt miteinander verbindet. Die Straße erwies sich eher als materielle Metaphorik. Sie soll hier deshalb als historisch gewachsener Diskurs und als Symbol verstanden werden, das sowohl für bürgerliche Alltagsöffentlichkeit als auch für politische Widerständigkeit und gegenbürgerliche Randständigkeit steht. Wo inszenieren sich Punks schließlich selbstverständlicher als auf der Straße?

Die Straße ist für Richard Sennett darüber hinaus „ein Synonym für Provokation und Anstöße, welche die Stadt bereithält, Provokation, die überwiegend von Erfahrungen mit dem Unerwarteten herrühren" (1997: 214). Und das Unerwartete geschieht im heterogenen Straßenmilieu besonders häufig. Hier treffen Individuen aus unterschiedlichen soziokulturellen Gruppen aufeinander, flanieren, observieren und inszenieren sich, interagieren und stiften dadurch gesellschaftliche Wirklichkeit.

„Es ist schon paradox: Die Straße ist ein offenes Buch und zugleich ein Geheimnis. Es gibt keinen gesellschaftlichen Raum der vergleichbar heterogen oder handlungsreich ist, [...] der von jedem Bürger betreten, beeinflusst und auf diese Weise mitgestaltet werden kann.“ (Geschke 2009: 11).

In der Entstehungsphase des Bürgertums Mitte des 19. Jahrhunderts war die Straße der Raum zum Flanieren (Benjamin 1983). In diesem Sinne argumentiert auch Therese Schütz:

„Der Straßenraum liegt zwischen den einzelnen Orten und der gesamten Stadt, gleichzeitig eingebettet und vermittelnd mit ihrem Kontext. Ineinander, untereinander, übereinander weben sich die Wege menschlichen Handelns zu einem dichten Stoff. Sein Muster zeigt Beziehungsgeflechte zwischen Gesellschaft, Gemeinschaft, spannt Brücken zwischen Vergangenheit und einer möglichen Zukunft. Im Mäandern der Straße durch die Stadt kristallisieren sich feingliedrige soziale Netze, ökonomische Abhängigkeiten, technische Infrastrukturen und rechtliche Rahmengerüste." (2013: 41)

Im Zuge der Modernisierung wurde die Straße schließlich zum „Schauplatz des modernen Lebens" (Sagner 2006: 13), zur Arena des bürgerlichen und damit gesellschaftlichen Selbstverständnisses und damit zur „Basis von Öffentlichkeit“ (Hoffmann-Axthelm 1997: 21). Die Straße wurde nun auch verstärkt zu einem konfrontativen Ort, an dem soziale Missstände sichtbar gemacht und politische Kämpfe ausgetragen wurden. Sie diente der performativen Selbstvergewisserung sowie der kulturellen und politischen Einflussnahme. Trotz dieser diskursiven Vereinnahmung durch das Bürgertum, haftet der Straße bis heute aber auch das Pedestrische, also das Niedere und Gewöhnliche an. Sie steht also nicht nur für die (bürgerliche) 
Gesellschaft, sondern auch für deren Ränder: Obdachlose, Prostituierte, Kriminelle - die Unangepassten und Unerwünschten. Demgegenüber sind auch Repräsentant_innen des Gesetzes oder anderer ordnender Diskurse Teil der Straße. Schließlich ist die Straße keine Tabula rasa soziokultureller Wirklichkeit, sondern in all ihren Erscheinungen und Akteur_innen ein Spiegel gesellschaftlicher Verhältnisse. Sie sei darüber hinaus ein metaphorischer Traum ihrer Potenzialität:

„Straße ist das schlechthin Mögliche: wo in der Tat, weil alle hindürfen, nichts auszuschließen ist. So dreht sich die Geschichte der Straße um die Quadratur des Kreises, universellen Verkehr zuzulassen und möglichst alles, was daraus an Zu- und Unfällen folgen könnte, auszuschließen. [...] Man erlebt nicht viel auf der Straße, aber man könnte etwas erleben, wenn man zu Fuß gehend sich auf die Straße einlässt. [...] Die Straße ist ein Raubtier, voller vermuteter Gefahr gerade dort, wo sie leer ist, und voller ängstigender und lockender Ausbruchmöglichkeiten da, wo sie vor Leben überquillt.“ (Hoffmann-Axthelm 1997: 15f.)

Im 19. Jahrhundert wurde der Städte- und Straßenbau des europäischen Westens grundlegend reformiert. Als erstes mussten die schmutzigen und dunklen Gassen von Paris unter dem Stadtplaner Georges-Eugène Haussmann lichten Promenaden weichen. Die Straßen wurden nun gepflasterte Verkehrswege, gesäumt von opulenten Häuserfronten, mit Renaturalisierungsinseln sowie Waren- und Konsumwelten. Ihre materielle Assemblage bestand zudem aus herausgeputzten Passant_innen, Straßenkünstler_innen, Fahrrädern und Autos sowie populärkulturellen Insignien. Nachts bot sie die Möglichkeit zur geduldeten und scheinbar kontrollierten Devianz von gesellschaftlichen Regeln in Kneipen, Kasinos oder auf dem Straßenstrich. Trotz bürgerlicher Normalisierungs- und Ordnungsfantasien bleibt der Straße bis heute ein Beigeschmack der Verruchtheit - sei es materiell durch den Abfall, den die Akteur_innen zurücklassen, sei es diskursiv-metaphorisch durch Devianzen.

Der kulturelle Mythos von der Eroberung der Straße durch die bürgerliche Ordnung zieht zudem einen weiteren nach sich, nämlich den der antibürgerlichen Rückeroberung, vor deren Hintergrund sich die Chaostage 1995 lesen lassen. Die Straße als Ort des Pedestrischen, des Niederen, bot schon vor den Chaostagen einen beliebten Identifikations- und Treffpunkt für Punks, die sich selbst auch (anti-)souverän als „Abfallprodukte der Gesellschaft“[3], als „Pöbel und Gesocks“[4] bezeichnen. Sowohl in ihrem Selbstbild als auch in der Fremdzuschreibung nehmen Punks die gesellschaftliche Position des ,Anderen“ ein. Dieses Bild konstituiert sich in der direkten Auseinandersetzung mit der Figur des zivilisierten, ruhe- und ordnungsliebenden Bürgers[5]. So werden mittels spezifischer Kleidungs- und Verhaltenscodes Lärm, Schmutz und Chaos rituell inszeniert. Dazu gehört der passive Protest, beispielsweise durch das Haarefärben genauso, wie aktive Angriffe auf die bürgerliche Ordnung der Straße.

Die Chaostage 1995 selbst begannen wie ein groß angelegtes Szenetreffen, bei dem bunt gekleidete Punks die Innenstadt Hannovers füllten, idealtypisch Bier tranken und laute Musik hörten. Dies erzeugte eine Spannung zum Rest der Bevölkerung. Den antibürgerlichen Punks begegneten die Bürger_innen mit Entsetzen, Unverständnis und Beschimpfungen. Die 
Störung der Ordnung - durch die symbolische und praktische Raumnahme der Punks - schien aufzugehen. An ihr entzündete sich durch eine Kette von Ereignissen sowie durch das Hinzutreten weiterer Akteur_innen der riot der Chaostage 1995.

Infolge der kurzen symbolischen Eroberung der Straße durch die Punks wurde die Polizei alarmiert und die Presse gerufen, um die ,Ordnung' wiederherzustellen. Aus dem Punktag mit Volksfestcharakter wurde ein Fest der Verwüstung und Gewalt: Die Straße war nun nicht mehr nur in ihrer symbolischen Bedeutung umkämpft, die Gehwege dienten nicht länger nur dem Feiern und Herumsitzen, ihre Schaufenster nicht mehr nur dem Kontrast zur Punkkultur. Mit einsetzender Gewalt wurden die öffentliche Mobilität und der Verkehr unmöglich - die Stadt wurde ,besetzt' und umkämpft. Die Polizei drängte die Punks in die sogenannte Nordstadt ab. Diese wiederum erkämpften sich durch Barrikaden, Wurfgeschosse und Körperkraft neuen Raum. Die materielle Grundlage ihres Kampfes entstammte der Straße selbst: Pflastersteine, Café-Mobiliar oder Zäune. Die Straße wurde also zum Austragungsort und zugleich zur Waffe des Straßenkampfes. Auf diese ,Neugestaltung' der Straße reagierte die Polizei entsprechend mit Einsatztruppen, Wasserwerfern und Räumfahrzeugen. Die Plünderung eines PennyMarkts durch Punks (und Bürger_innen) stellt im Zuge dieser Ereignisse die größte Annektierung und ,Umgestaltung 'bürgerlicher Infrastruktur dar. Die Straße entpuppte sich als „die einzige gesellschaftliche Bühne, auf der Krieg und Frieden noch life [sic!] und im Maßstab 1:1 gespielt werden" (HoffmannAxthelm 1997: 20f.). Auf der Straße vollzog sich zudem die Identitätsbildung der Akteur_innen (Mahnert 1997: 83).

Das (Zer-)Stören und Wiederherstellen der Ordnung gestaltete sich bei den Chaostagen 1995 also als eruptiver Gewaltakt. Vor dem Hintergrund der symbolischen Bedeutung der Straße entpuppte sich die - auf den ersten Blick sinnlos erscheinende - Gewalt als Kampf um Repräsentation und Macht. In der bürgerlichen Nachrichtenberichterstattung wurden die Geschehnisse auf der Straße intensiv verfolgt, bildhaft dokumentiert, aufbereitet und mit eigenem Sinn versehen. Austauschbare Bilder von brennenden Barrikaden, zerschlagenen Scheiben und Straßenschlachten mit der Polizei wiederholten sich ständig. Augenzeugen wurden zitiert und es wurde von „bürgerkriegsähnlichen Zuständen“ (Haase 2015) gesprochen, von „Feinde[n] der Zivilisation“ (Geiling 2000: 172), von einer „Gewaltorgie“ (NDR 2015). Die bürgerlichen Medien stilisierten den riot der Chaostage zum ,Anderen' der Gesellschaft. Dabei war diese Inszenierung selbst ein Affektspektakel, ihre Funktion die Normalisierung. So wurden der riot und die Punks als ,anormal' ausgestellt. Ihr Auftreten, ihre Parolen und ihre Gewalt verschmolzen im bürgerlichen Spektakel zu einem obszönen und kohärenten Bild des Unmoralischen.

„Im Fall der Denormalisierung wird das Plötzliche und Unerwartete betont und der leere Moment des Abbruchs der Normalität durch Wiederholung gefüllt. Dies geschieht sowohl durch eine Serie von Statements, in denen Betroffene und Augenzeugen den unfasslichen und unverarbeiteten Augenblick beschreiben, als auch durch mehrmaliges Wiederholen von spektakulären Momentaufnahmen [...].“ (Thiele 2007: 109) 
In Abgrenzung zu diesem Bild wurde es dem bürgerlichen Publikum möglich, sich seiner Normalität und damit seiner selbst zu versichern. Der Denormalisierung auf der Straße folgte eine Renormalisierung im Fernsehen. Das Medienspektakel kannte dabei keine Ambivalenzen: Die Selbstbeschreibungen der Punks und ihre Parolen auf Flyern und Plakaten, zum Beispiel die Ankündigung der Chaostage als „,Hate Parade““ (Graf 2003: 130), wurden trotz ihrer Referenzialität, ihrer ironischen Semantik und ihrer Überspitzungen zu Wahrheiten gemacht. Die in Ansätzen souveränen Aneignungsversuche des Spektakels vonseiten der Punks wurden übersehen oder gingen in ihm auf. Parolen wie „Presse befiehl, wir folgen!“[6] verdeutlichen den ironischen Umgang mit der Berichterstattung. Die Flugblätter und Plakate trugen also nicht nur zur konkreten Gestaltung des riots bei, sondern luden auch die Symbolik der Straße mit auf. Dennoch schaffte es die Inszenierung der Punks nicht, über die (ironische) Überaffirmation ihrer Rolle als ,anormal' hinauszuweisen. Wie zuvor beschrieben, bedarf das Spektakel der Normalisierung durch diese inszenierte Eindeutigkeit. Und da die Punks diesen Dualismus bestätigten, wurden sie zur Zielscheibe für das Normalisierungsspektakel. Zwischen Medien und Straße entwickelt sich zwar eine eigene Dynamik, die sich gegen die Ordnung richtete, sie stellte jedoch keinen Angriff auf die normalisierende (objektive) Gewalt dar. Die Deutung des Geschehens durch die Punks trat hinter jener der bürgerlichen Gesellschaft zurück.

\section{Die Weiterführung der Chaostage in den Medien: Die Mediatisierung des riots}

Weil Punk provozieren möchte, sucht er die Nähe zu Medien und Presse. Die Wechselwirkungen mit den bürgerlichen Medien machen auch die Dynamik und die Qualität der Chaostage als medialen riot aus. So hat die Berichterstattung über die Chaostage 1994 zu einer Intensivierung der Krawalle im Jahr 1995 geführt (Nagenborg 1999). Die Medien schrieben dem Punk das „Merkmal ,Gewalt““ (1999: 7) zu, was „Spiritus Rector“[7] online folgendermaßen kommentierte: „,Die Medien scheinen sich ... für gar keine andere mögliche Entwicklung der Chaos-Tage zu interessieren.“' (zit. nach Nagenborg 1999: 8). Dies spitzt Spiritus Rector auf die Formel „Journalisten sind Mörder!“ zu und stellt abschließend fest: „Journalisten sind unsere Freunde!“ (Spiritus Rector 1996a). Seine Provokation liegt auf der Hand: Einerseits kritisiert er die Position der Medien, indem er Journalist_innen selbst zu Täter_innen stilisiert, andererseits suggeriert er ein gemeinsames Interesse und eine opportune Freundschaft.

Für die mediale Strategie der Punks war es zentral, die Bildberichterstattung neu zu besetzen und umzudeuten. Hierfür ist es hilfreich im Kopf zu behalten, dass Interpretationen immer in einem sozialen Kontext vorgenommen werden und somit auch die Objektivität von journalistischen Bildern illusorisch ist: „Die Wahrheit ist Bild, doch es gibt kein Bild von Wahrheit“ (Latour 2002: 10). Ein anti-essentialistischer Blick auf Abbildungen, macht die vielfältigen Anschlüsse sichtbar, die stets Abbild, aber nicht real sind.

Die antibürgerliche Medienstrategie der Punks bestand also in Dekontextualisierung, Satire, Überspitzung und Übertreibung. Mit dem Ziel, eine bereits vorhandene Hysterie weiter anzufachen, wurde die mediale Narrationen 
überaffimiert und ironisiert. So lud etwa der (fiktive) Bund schlesischer Freizeitmaler mit einem Flugblatt dazu ein, „malerische Ausblicke“ mit „pittoresken Punker[n]“ (Chaostage.de 1995a) zu zeichnen. Die Deutsche Gesellschaft für Chaosforschung kündigte ein „Feldforschungsprojekt“ an und lud zu einem „Punkimmitationslehrgang“ (Chaostage.de 1995f). Die „Schutt-und-Asche“-Rhetorik wurde durch die Ankündigung, ,Atombomben“ und „C-Waffen“ einzusetzen bedient (Chaostage.de 1995b) und überspitzt: „Wir werden nämlich genug davon mitbringen!“ (Chaostage.de 1995g). Daneben wurden kritische Stimmen laut, die von den Chaostagen mehr erwartet hatten als bloße Krawalle gegen Polizei und Bürgertum (Chaostage.de 1995c). So wurde in die „bunteste Stadt“ geladen, zu „Bands (hoffentlich!)“. Es lockten „[a]n jeder Ecke: Aktionen“ (Chaostage.de 1995d), auch wenn diese nur in „Bullenverarschung“ (Chaostage.de 1995e) bestünden.

Besonders hervorzuheben ist in diesem Zusammenhang die Mediencollage Kampf der Welten: Chaostage 1995, die als restaurierte DVD-Fassung 2006 und durch Streaming größere Verbreitung fand (Sprengkopf Recklinghausen 1996). Die filmische Collage verknüpft Fotos, Dokumentar- und Spielfilmszenen, Nachrichtensendungen und Statements verschiedener Akteur_innen. Der Film entfaltet seine Wirkung aus der De- und Rekontextualisierung der Berichterstattung und zielt so auf bürgerliche Hysterie und Kriegsangst. Diese experimentellen Assoziationen von Straßenunruhen und -dynamiken mit verschiedenen anderen Materialien erzeugt im Ergebnis eine neue Stufe von Chaos. Der riot war damit nicht mehr nur Teil der medialen Berichterstattung, er erfasste diese und wendete sie gegen sich selbst.

Der Filmtitel spielt auf H. G. Wells Roman Krieg der Welten an. Eine Alien-Invasion führt dort dazu, dass die Menschheit (beinahe) zugrunde geht und in Apathie, Leid und Elend verfällt. Szenen aus der Verfilmung werden mit Einspielern über die Ereignisse in Hannover unterlegt. Weiterhin werden Anmoderationen mehrerer Fernsehsendungen gezeigt, in denen fragend und ratlos mit dem Phänomen der Chaostage umgegangen, Hannover mit „Beirut oder Belfast“ verglichen oder folgende Einordnung versucht wird: „,Den Bullen eins auf'e Fresse geben, Scheiben klirren lassen, und einmal mit der grünen Minna fahren.' Das ungefähr ist die intellektuelle Ausgangsposition der Punker für ihre Chaostage. "Eine bürgerliche Interpretation der Krawalle scheint hier unmöglich, stattdessen wird ihr Sinn von Protagonist_innen erläutert: „Spaß, der heißt: Saufen, kaputtmachen, sich mit den Bullen anlegen, das heißt das“, führt beispielsweise der Sänger einer bekannten Punkband aus und fährt fort: „Ihr versteht das nicht. Das kommt einfach aus mir raus und dann mach' ich alles kaputt. Punkt. Hast du schon jemals eine Mülltonne durch ein Schaufenster geschmissen? Das macht einfach Bock. Brauchst'e kein Hooligan für sein. Ich bin Punkrocker und schmeiß’ Mülltonnen.“

Der Film verdeutlicht zudem extreme Aversionen der Bürger_innen: „Die? Die gehören alle unter die Erde!“, „Früher hätte man die ..." und so weiter. Der bürgerliche Affekt und die Ratlosigkeit zeugen von der Sehnsucht nach ,Ordnung' und der, guten alten Zeit'. Diese moderne Passion des ,Ordnungsmachens' legt offen, dass Ambivalenzen ein Tabu sind (vgl. Bauman 1992). An die Äußerungen schließen die Filmemacher_innen mit einer Vielzahl (ver-) störender Bilder an, die unter anderem Konzentrationslager und Massenerschießungen im Zweiten Weltkrieg zeigen. Die „Forderung, daß Auschwitz 
nicht noch einmal sei“ (Adorno 1971 [1966]: 88), droht durch die Äußerungen der Bürger_innen in Verbindung mit diesen Bildern unerfüllt zu bleiben

Die mediale Berichterstattung der Chaostage erzeugte insgesamt eher einen Sinnzusammenbruch, eine ,Kontingenzauflösungsstörung', anstatt eine Erklärung für die Ereignisse zu liefern. Sie blieben als Phänomen medial unzugänglich und richteten sich als riot gegen die Gesellschaft als Ganzes, indem sie deren Wertvorstellungen negierten. Sie richteten sich auch gegen das Spektakuläre der Gesellschaft, gegen das normalisierende Spektakel auf der Straße und im Fernsehen, in welchem objektive Gewalt durch mediale Disziplinierung ausgeübt wurde. Der Film führt außerdem die Gewaltfantasien ,aus der Mitte als strukturell und latent faschistische Ordnungsliebe vor. So zeigt die Collage beispielsweise einen Kommentar der ARD:

„Gestatten Sie mir aus der Ratlosigkeit einen Erklärungsversuch, nämlich den, dass der Mensch nicht gut ist [...]. Es gibt das Böse und das lässt sich weder von Soziologen noch von Politikern wegbehandeln, dummerweise auch nicht wegprügeln, so sehr ich persönlich mir es auch manchmal wünsche. In dieser Gesellschaft gilt die Entfaltung des Einzelnen als höchstes Ziel; da entfaltet sich viel Gutes, aber eben auch Böses. Das lässt sich mit Mitteln staatlicher Gewalt nicht beseitigen, aber eingrenzen. Geschieht das nicht, gibt es am Ende keine Gesellschaft mehr, die diesen Namen verdient. [...] [W]enn ich ganz grundsätzlich abzuwägen habe zwischen einerseits dem Recht, sich die Haare grün zu färben und sich eine Sicherheitsnadel durch die Backe zu stechen, und andererseits dem Recht des Volkswagenbesitzers in Hannover darauf, dass ihm nicht Idioten seinen Golf zerkloppen, entscheide ich, auch wenn das im Einzelfall ungerecht ist, gegen das Recht auf grüne Haare.“

Nicht nur die Chaostage selbst, sondern auch die Flyer und der Film Kampf der Welten sorgten für Unruhe. Der riot fand also nicht nur auf materieller, sondern auch auf medialer Ebene statt. Ausgangspunkt unserer Interpretation ist hier die Ambiguität der karnevalesken Bilder: Die ambivalenten Bilder der Chaostage führten demnach zu einem Sinnzusammenbruch, einem iconoclash [8], der sich in der in der Weiterführung der riots , mit anderen Mitteln' verdoppelte. Die bürgerlichen Moral- und Wertvorstellungen wurden mit ihren sinnlogischen Konsequenzen konfrontiert und die Differenz zwischen bürgerlicher Moral und dem, Anormalen ' gewaltvoll eingeebnet.

Hierbei arbeiteten die rioter mit verschiedenen Mitteln der Kommunikationsguerilla (zur Übersicht: Schölzel 2013). Diese „ist Teil eines Prozesses, in dem gesellschaftliche Herrschaftsverhältnisse kritisiert und angegriffen werden" (Blissett/Brünzels 1997: 6). Im Kontext der Chaostage richtete sich die Kritik auf gesellschaftliche Hegemonien, was unter anderem auf den Flyern sichtbar wird: Einige griffen bewusst die mediale Rhetorik (etwa die „Schutt-und-Asche“-Legende) auf und ironisierten oder radikalisierten sie, indem sie - wie im einleitenden Zitat - dazu aufforderten „Hannover zur Wiese“ zu machen. Gerade im Film Krieg der Welten wird den Rezipient_ innen eine bis dahin verborgene Kontingenz vor Augen geführt: „Es geht weniger um Gegeninformation als darum, das Rezeptionsverhalten und den Umgang der ZuhörerInnen mit den massenmedialen ,Informationen' zu verändern“"(autonome a.f.r.i.k.a. gruppe/mittlerer neckar 1994: 146). So soll die 
Collagierung der Medienfragmente nicht entwertend oder zerstörend wirken, sondern ist eine Aneignungsstrategie, die neue Interpretationsräume eröffnen kann. Diese Praxis wendete sich explizit gegen die Medien als Herrschaftsmittel:

„Kommunikationsguerilla ist politische Militanz. Sie unterscheidet sich insofern von der herkömmlichen (autonomen) Militanz, als sie die Codes von Macht und Herrschaft nicht einfach zerstört, sondern zu entstellen versucht und ihnen damit ihre Wirksamkeit nehmen will. [...] Ziel ist weder die Unterbrechung noch die permanente Aneignung eines Kommunikationskanals, sondern die Entwendung und Entstellung der transportierten Botschaften.“ (autonome a.f.r.i.k.a.-gruppe 1998)

Den Modus einer solchen „Entwendung und Entstellung“ hat Sofsky (1996) als Gewalt gegen die Differenzen der Symbole bezeichnet. Herrschende Symbole würden hierbei umgedeutet und entfremdet.

Der riot der Chaostage 1995 richtete sich aber auch auf einer materiellen Ebene gegen die bürgerliche Ordnung und wurde so Teil eines normalisierenden Spektakels, welches auf beiden Seiten selbstversichernd wirkte: Für das Bürgertum funktionierte das Spektakel einseitig gesellschaftsstabilisierend, während die rioter medial versuchten, die Differenzierung zwischen bürgerlicher Ordnung und ihrem ,Anderen' aufzuheben. Die Frage, wer diese Differenz ziehen und definieren darf, wurde neu gestellt: Der riot war - in diesem Sinne - ein „Aufstand der Zeichen“ (Baudrillard 1978), ein Angriff auf „diese Semiokratie“ (ebd.: 23). Diese Baudrillard'sche Vorstellung von riot ist darüber hinaus mit dem Städtischen verknüpft: „Die Matrix des Urbanen ist [...] die [...] Realisierung einer Differenz (der Operation des Zeichens)“ (1978: 20). Das Zeichensystem der,Ordnung ' wird durch den riot als Ganzes angegriffen und seine Fragilität zur Schau gestellt.

\section{Schluss}

In unseren Ausführungen haben wir das Ereignis der Chaostage 1995 hinsichtlich ihrer Wirkungen als riot untersucht. Gewalt interpretierten wir im Kontext der bürgerlichen Gesellschaft und der Punks als gesellschaftliche Relation und Distinktion. Es wurde deutlich, wie die Chaostage auf zwei Arenen zugriffen: Im urbanen Raum, auf der Straße, entstand ein riot, der sich in seinem Verlauf spielerisch zu einer symbolisch-materiellen Raumnahme und einem Normalisierungsspektakel entwickelte. Die Teilnehmer_innen der Chaostage hielten der Gesellschaft einen Spiegel vor, wodurch ihre bürgerlichen Grundwerte, derer sie sich sicher geglaubt, entzaubert wurden. Während sich die Gewaltausübung der Punks gegen die (bürgerliche) Ordnung und die Gesellschaft als Ganzes richtete, wendete sich die Gewalt der bürgerlichen Gesellschaft ordnend gegen den riot, ihr Selbstverständnis zugleich normalisierend. Die bürgerliche Gesellschaft inszenierte ein politisches Spektakel, in dem der Aufstand in den - von den Medien geschürten - Erwartungen aufzugehen schien.

Indem der riot medial wurde, störten die Punks die öffentliche Ordnung auf einer anderen Ebene und riefen eine mediale Hysterie hervor. Die Dekontextualisierung der Medienmotive und -zitate zeigte die prinzipielle 
Kontingenz medialer Berichte auf. Auf dieser Ebene richtete sich die Gewalt gegen die hegemoniale Differenz der Zeichen und gegen die Ordnung der Gesellschaft.

Auch wenn das Ereignis mehr als zwanzig Jahre zurückliegt, lässt sich anhand der Chaostage die enge Verknüpfung von riot, Gewalt und Spektakel verdeutlichen. Dadurch wird es über eine Analyse der Chaostage hinaus möglich, andere ungerichtete urbane Gewaltausbrüche hinsichtlich ihrer medialen und transformativen Eigenschaften zu untersuchen: Es werden Wirkmechanismen scheinbar sinnloser Gewalt auf der Straße sichtbar und die ihr strukturell vorgängigen sowie potenziell folgenden Gewaltformen. Von einer noch ausstehenden systematischen Betrachtung der Chaostage von 1995 erhoffen wir uns ein besseres Verständnis für reziproke Dynamiken zwischen Gesellschaft und scheinbar sinnfreien riots, das geeignet ist, auch die immanenten Positionen der marginalisierten rioter aufzuzeigen.

\section{Endnoten}

[1] Gewalt stellt bei den englischen riots von 2011 beispielsweise ein Hindernis für linke Solidarisierungen dar (Hoekmann 2012: 123).

[2] Eine methodische Anmerkung: Unsere Ausführungen versuchen einen theoretischen Sachverhalt an einem empirischen Ereignis nachzuvollziehen. Es kommt uns daher nicht auf eine strikte empirische Beweisführung an, sondern auf die konzeptionelle Nachvollziehbarkeit. Das Anschauungsmaterial zu den Chaostagen ist im Quellenverzeichnis aufgeführt. Eine systematische und methodologisch robuste Analyse können wir an dieser Stelle nicht leisten. Da allerdings ersichtlich geworden ist, dass eine sozialwissenschaftliche Auseinandersetzung mit den Chaostagen bisher kaum erfolgt ist, betrachten wir diese Vorgehensweise als legitim und sinnvoll, um eine Lücke in der Aufarbeitung zu schließen. Wir verweisen auf das Material unter Angabe seiner Fundorte im Internet. Der angeführte Film ist - zumindest vorläufig - unter der angegebenen Web-Adresse abrufbar. Unsere Auswertungen beziehen sich auf die ersten 15 Minuten des Films und unsere Angaben erfolgen daher ohne konkrete Zeitstempel.

[3] Song- und Albumtitel der (DDR-)Punkband Schleim-Keim (neu aufgelegt 1992).

[4] Song- und Albumtitel (sowie nach Rechtsstreitigkeiten mit einer Brauerei auch Bandname) der Punkband Beck's Pistols (1990).

[5] Bewusst geschieht an dieser Stelle keine geschlechtersensible, grammatikalische Angleichung, da die historisch-diskursive Figur des Bürgers, auf die wir uns beziehen, sich selbst über Männlichkeit konstituiert und diese als absolut setzt.

[6] Diese Aussage wird häufig zitiert, ohne dass eine eindeutige Quelle auffindbar ist.

[7] Im Untertitel der (archivierten) Homepage findet sich eine Selbstdarstellung als „geistige[r] Brandstifter“, gefolgt von der Forderung: „Die menschliche Zivilisation muss vernichtet werden! Sofort!“ (Spiritus Rector o. J.). Auf chaostage.de (o. J.) wurde die Aufgabe von Spiritus Rector sowie seines Internetauftritts Cannibal Home Channel als „Informationsvergiftung“ aufgefasst, welche „fast täglich blutige Parolen frei Haus“ geliefert hätte, die „von den Medien nur zu gerne aufgegriffen“ worden wären. Somit sei den bürgerlichen Medien „Ihr [sic!] eigener Sensationsjournalismus rektal eingeführt" worden. Bei den Chaostagen handelte es sich um einen der ersten Proteste, die das Internet nutzten. Dieses war bereits so weit verbreitet, dass es als Infrastruktur zur Mobilisierung wie auch zur Herstellung einer gewissen Gegenöffentlichkeit verwendet werden konnte, wie insbesondere das hier herangezogene Archiv chaostage.de belegt.

[8] Bruno Latour hat auf die Unterscheidung zwischen „Ikonoklasmus“ und „Ikonoclash“ hingewiesen. Ersterer sei ein Bildersturm, welcher im Akt des Zerstörens eines Bildes entstehe. Die Zerstörung sei in diesem Falle sinnhaft und phänomenologisch greifbar. Der „Ikonoclash“ hingegen irritiere, da die Zerstörung nicht eindeutig (be-)greifbar sei und kontingent scheine. 


\section{Autor_innen}

Yannick Kalff ist Soziologe mit den Schwerpunkten Arbeits-, Organisationssoziologie, Krise der Arbeit, alternative Ökonomien und Selbstorganisation.

yannick.kalff@uni-jena.de

Katharina Warda ist Kultursoziologin und Literaturwissenschaftlerin. Ihre Interessen sind Postcolonial- und Genderstudies, Literatur des 20./21. Jh. sowie des Internets.

katharina.warda@fu-berlin.de

\section{Literatur}

Adorno, Theodor W. (1971 [1966]): Erziehung nach Auschwitz. In: Gerd Kadelbach (Hg.), Erziehung zur Mündigkeit. Vorträge und Gespräche mit Hellmut Becker 1959-1969. Frankfurt am Main: Suhrkamp, 88-104.

autonome a.f.r.i.k.a.-gruppe (Hg.) (1998): Vorsprung durch Technik? Internethype, Gegenöffentlichkeit und Widerstand. Vernetzung \& Kommunikationsguerilla. http://kguerilla. org/sites/default/files/vorsprung-durch-technik.pdf (letzter Zugriff am 26.1.2016).

autonome a.f.r.i.k.a.-gruppe mittlerer neckar (Hg.) (1994): Kommunikationsguerilla - Der Kampf geht weiter. Anstiftung zu einer subversiven kommunikativen Praxis. In: autonome a.f.r.i.k.a.-gruppe/mittlerer neckar (Hg.), Medienrandale. Rassismus und Antirassismus. Die Macht der Medien und die Ohnmacht der Linken? Grafenau: Trotzdem, 143-161.

Baacke, Dieter (1985): Jugendliche Lebensstile. Vom Rock'n'Roll bis zum Punk. In: Bildung und Erziehung 38/2, 201-212.

Bartz, Christina / Krause, Marcus (2007): Einleitung: Spektakel der Normalisierung. In: Christina Bartz / Marcus Krause (Hg.), Spektakel der Normalisierung. München: Wilhelm Fink Verlag, 7-24.

Baudrillard, Jean (1978): Kool Killer. oder Der Aufstand der Zeichen. Berlin: Merve Verlag.

Bauman, Zygmunt (1992): Moderne und Ambivalenz. Das Ende der Eindeutigkeit. Hamburg: Junius.

Benjamin, Walter (1983): Das Passagen-Werk. Frankfurt am Main: Suhrkamp.

Bennett, Andy (2006): Punk's not Dead. The Continuing Significance of Punk Rock for an Older Generation of Fans. In: Sociology 40/2, 219-235.

Blissett, Luther / Brünzels, Sonja (1997): Handbuch der Kommunikationsguerilla. Jetzt helfe ich mir selbst. Hamburg: Verlag Libertäre Assoziation.

Chaostage.de (o. J.): Flashback. http://www.chaostage.de/a/home/p/flashback.php (letzter Zugriff am 1.2.2016).

Chaostage.de (1995a): Bund schlesischer Freizeitmaler. http://www.chaostage.de/ downloads/flyer/1995/freizeitmaler.pdf (letzter Zugriff am 1.2.2016).

Chaostage.de (1995b): „Schutt und Asche“? http://www.chaostage.de/downloads/ flyer/1995/atombomben.pdf (letzter Zugriff am 1.2.2016)

Chaostage.de (1995c): Chaos-Tage ,95 Ein gutes Argument gegen die Expo! http://www. chaostage.de/downloads/flyer/1995/anregung.pdf (letzter Zugriff am 1.2.2016).

Chaostage.de (1995d): Komm auch du in die bunteste Stadt der Welt! http://www.chaostage. de/downloads/flyer/1995/bunteste.pdf (letzter Zugriff am 1.2.2016).

Chaostage.de (1995e): Wochenend-Ausflug in eine fremdartige Stadt. http://www. chaostage.de/downloads/flyer/1995/bullenverarschung.pdf (letzter Zugriff am 1.2.2016).

Chaostage.de (1995f): Deutsche Gesellschaft für Chaosforschung. http://www.chaostage. de/downloads/flyer/1995/chaosforschung.pdf (letzter Zugriff am 1.2.2016).

Chaostage.de (1995g): Schutt und Asche? Könnt ihr haben! Und zwar Säckeweise! http:// www.chaostage.de/downloads/flyer/1995/mitbringen.pdf (letzter Zugriff am 1.2.2016).

Cuevas, Minerva (2008): Patria. In: Sønke Gau / Katharina Schlieben (Hg.), Spektakel, Lustprinzip oder das Karnevaleske? Ein Reader über Möglichkeiten, Differenzerfahrungen und Strategien des Karnevalesken in kultureller / politischer Praxis. Berlin: b-books Verlag, 289-294.

Debord, Guy (1996): Die Gesellschaft des Spektakels. Berlin: Edition Tiamat. 
Dubet, François (1997): Die Logik der Jugendgewalt. Das Beispiel der französischen Vorstädte. In: Trutz von Trotha (Hg.), Soziologie der Gewalt. Opladen: Westdeutscher Verlag, 220-234.

Dzudzek, Iris / Müller, Michael (2013): Der Lärm des Politischen. Die Londoner riots 2011 und ihre politischen Subjekte. In: s u b / u r b a n. zeitschrift für kritische stadtforschung $1 / 2,17-40$.

Eisenberg, Götz / Gronemeyer, Reimer (1993): Jugend und Gewalt. Der neue Generationenkonflikt oder der Zerfall der zivilen Gesellschaft. Reinbek bei Hamburg: Rowohlt.

Farin, Klaus (1997): Jugendkulturen zwischen Kommerz \& Politik. Erfurt: Landeszentrale für politische Bildung, Thüringen.

Farin, Klaus / Seidel, Eberhard (2012): Krieg in den Städten. Jugendgangs in Deutschland. Berlin: Archiv der Jugendkulturen Verlag.

Foucault, Michel (1974): Die Ordnung der Dinge. Eine Archäologie der Humanwissenschaften. Frankfurt am Main: Suhrkamp.

Fukuyama, Francis (1992): The end of history and the last man. London: Penguin.

Gabbert, Wolfgang (2004): Was ist Gewalt? Anmerkungen zur Bestimmung eines umstrittenen Begriffs. In: Julia M. Eckert (Hg.), Anthropologie der Konflikte. Georg Elwerts konflikttheoretische Thesen in der Diskussion. Bielefeld: transcript, 88-101.

Gau, Sønke / Schlieben, Katharina (2008): Spektakel, Lustprinzip oder das Karnevaleske?. Über Möglichkeiten, Differenzerfahrungen und Strategien des Karnevalesken in kultureller / politischer Praxis. In: Sønke Gau / Katharina Schlieben (Hg.), Spektakel, Lustprinzip oder das Karnevaleske? Ein Reader über Möglichkeiten, Differenzerfahrungen und Strategien des Karnevalesken in kultureller / politischer Praxis. Berlin: b-books Verlag, 9-30.

Geiling, Heiko (1995): „Chaos-Tage“ in Hannover. Vom Ereignis zum Mythos. In: Vorgänge $132 / 4,1-6$

Geiling, Heiko (2000): Punk als politische Provokation. Mit den Chaos-Tagen in Hannover zur Politik des ,gesunden Volksempfindens'. In: Roland Roth / Dieter Rucht (Hg.), Jugendkulturen, Politik und Protest. Vom Widerstand zum Kommerz? Opladen: Leske + Budrich, 165-182.

Geschke, Sandra Maria (2009): Straße als kultureller Aktionsraum - eine Einleitung. In: Sandra Maria Geschke (Hg.), Straße als kultureller Aktionsraum. Interdisziplinäre Betrachtungen des Straßenraumes an der Schnittstelle zwischen Theorie und Praxis. Wiesbaden: VS Verlag für Sozialwissenschaften, 10-30.

Graf, Christian (2003): Punk! Das Lexikon. Berlin: Schwarzkopf \& Schwarzkopf.

Haase, Bernd (2015): Als die Chaostage Hannover erschütterten. http://www.haz.de/ Hannover/Aus-der-Stadt/Uebersicht/Mit-aller-Gewalt-Chaostage-erschuetternHannover (letzter Zugriff am 1.2.2016).

Hafeneger, Benno / Stüwe, Gerd / Weigel, Georg (1993): Punks in der Großstadt - Punks in der Provinz. Projektberichte aus der Jugendarbeit. Opladen: Leske + Budrich.

Hahn, Anne (2009): Pogo im Bratwurstland. Punk in Thüringen. Erfurt: Landeszentrale für politische Bildung, Thüringen.

Hahn, Anne (2013): Disteln mit spitzen Zacken dran. Punk. Erfurt: Landeszentrale für politische Bildung, Thüringen.

Harvey, David (2012): Rebel cities. From the right to the city to the urban revolution. London: Verso.

Herbertz, Oliver (2011): Die Organisation von Chaostagen. Analyse zur Konstruktion von Objektivität. In: Gregor Betz / Ronald Hitzler / Michaela Pfadenhauer (Hg.), Urbane Events. Wiesbaden: VS Verlag für Sozialwissenschaften, 245-260.

Hoekman, Gerrit (2012): England 2011. Aufstand in den Suburbs. In: Wolf Wetzel (Hg.), Aufstand in den Städten. Krise, Proteste, Strategien. Münster: Unrast-Verlag, 109-125.

Hoffmann-Axthelm, Dieter (1997): Straße. Ritus und Aufruhr. In: Ästhetik \& Kommunikation 28/99, 15-20.

Inhetveen, Katharina (1997): Gesellige Gewalt. Ritual, Spiel und Vergemeinschaftung bei Hardcorekonzerten. In: Trutz von Trotha (Hg.), Soziologie der Gewalt. Opladen: Westdeutscher Verlag, 235-260.

Köster, Petra F. (2014): Punk - Kultur des Protests. In: Udo Fleck (Hg.), Neues Trierisches Jahrbuch. Trier: Selbstverlag, 83-95.

Latour, Bruno (2002): Iconoclash. Oder gibt es eine Welt jenseits des Bilderkrieges? Berlin: Merve-Verlag. 
Lau, Thomas (1992): Die heiligen Narren. Punk 1976-1986. Berlin u. a.: Walter de Gruyter.

Liebig, Steffen (2014): Soziale Unruhen als nicht-normierte Konflikte. Das Beispiel der englischen Riots von 2011. In: PROKLA. 44/175, 271-287.

Mahnert, Franz (1997): Von der ewigen Wiederkehr des Verdrängten. Sehnsucht nach der ,Rückkehr der Straße?‘. In: Ästhetik \& Kommunikation 28/99, 82-87.

Malott, Curry / Peña, Milagros (2004): Punk Rockers‘ Revolution. A Pedagogy of Race, Class, and Gender. New York: Peter Lang.

Marcus, Greil (1992): Lipstick traces. Von Dada bis Punk - kulturelle Avantgarden und ihre Wege aus dem 20. Jahrhundert. Hamburg: Rogner \& Bernhard.

Meinert, Philipp / Seeliger, Martin (2013): Punk in Deutschland. Sozial- und kulturwissenschaftliche Perspektiven. In: Philipp Meinert / Martin Seeliger (Hg.), Punk in Deutschland. Sozial- und kulturwissenschaftliche Perspektiven. Bielefeld: transcript, 9-55.

Metz, Markus / Seeßlen, Georg (2012): Kapitalismus als Spektakel. Berlin: Suhrkamp Verlag.

Nagenborg, Michael (1999): Gewalt in den Medien. Mit Gewalt Schlagzeilen machen Rückkopplungseffekte von dokumentarischen Gewaltdarstellungen am Beispiel ,ChaosTage‘. http://www.michaelnagenborg.de/pdf/gewalt_in_den_medien-anhang2.pdf (letzter Zugriff am 1.2.2016).

NDR (2015): Vor 20 Jahren: Die letzten Chaostage in Hannover. https://www.ndr.de/ kultur/geschichte/Vor-20-Jahren-die-letzten-Chaostage-in-Hannover,chaostage176. html (letzter Zugriff am 1.2.2016).

Nedelmann, Birgitta (1997): Gewaltsoziologie am Scheideweg. Die Auseinandersetzungen in der gegenwärtigen und Wege der künftigen Gewaltforschung. In: Trutz von Trotha (Hg.), Soziologie der Gewalt. Opladen: Westdeutscher Verlag, 59-85.

Pettenkofer, Andreas (2010): Radikaler Protest. Zur soziologischen Theorie politischer Bewegungen. Frankfurt am Main u. a.: Campus Verlag.

Pettenkofer, Andreas / Liell, Christoph (2004): Kultursoziologische Perspektiven in der Gewaltforschung. In: Christoph Liell / Andreas Pettenkofer (Hg.), Kultivierungen von Gewalt. Beiträge zur Soziologie von Gewalt und Ordnung. Würzburg: Ergon Verlag, 9-40.

Pfaller, Robert (2002): Die Illusionen der anderen. Über das Lustprinzip in der Kultur. Frankfurt am Main: Suhrkamp Verlag.

Reemtsma, Jan Philipp (2006): Die Natur der Gewalt als Problem der Soziologie. In: Mittelweg 36 15/5, 1-25

Reimitz, Monika (1989): Drinnen und Draußen. Vom Wohnen der Punks. In: Marlene Bock / Monika Reimitz / Horst-Eberhard Richter / Wolfgang Thiel / Hans-Jürgen Wirth (Hg.), Zwischen Resignation und Gewalt. Jugendprotest in den achtziger Jahren. Opladen: Leske + Budrich, 103-109.

Sabin, Roger (Hg.) (1999): Punk Rock: So What? The Cultural Legacy of Punk. London u. a.: Routledge.

Sagner, Karin (2006): „Die Eroberung der Straße begann im 19. Jahrhundert“. In: Karin Sagner / Matthias Amann (Hg.), Die Eroberung der Straße. Von Monet bis Grosz. München: Hirmer, 12-21.

Schölzel, Hagen (2013): Guerillakommunikation. Genealogie einer politischen Konfliktform. Bielefeld: transcript.

Schütz, Therese (2013): Auf der Straße gehen / Auf die Straße gehen. In: dérive - Zeitschrift für Stadtforschung 14/50, 37-44.

Schwell, Alexandra (2005): Anarchie ist die Mutter der Ordnung. Alternativkultur und Tradition in Polen. Münster: LIT.

Seifert, Jürgen (1996): Chaos-Tage: Modell Hannover. Aufenthaltsverbote als Instrument zur Durchsetzung von Versammlungsverboten? In: Kritische Justiz 29/3, 356-361.

Sennett, Richard (1997): Fleisch und Stein. Der Körper und die Stadt in der westlichen Zivilisation. Frankfurt am Main: Suhrkamp.

Soeffner, Hans-Georg (1992): Stil und Stilisierung. Punk oder die Überhöhung des Alltags. In: Hans-Georg Soeffner (Hg.), Die Ordnung der Rituale. Frankfurt am Main: Suhrkamp, 76-101.

Sofsky, Wolfgang (1996): Traktat über die Gewalt. Frankfurt am Main: S. Fischer.

Spiritus Rector (o. J.): Archiv der Homepage auf www.chaostage.de. http://www.chaostage. de/archiv/sites/1996_chc/deutsch/sr/index.html (letzter Zugriff am 1.2.2016).

Spiritus Rector (1996a): Journalisten sind Mörder. http://www.chaostage.de/archiv/ sites/1996_chc/deutsch/sr/artikel/moerder.html (letzter Zugriff am 1.2.2016). 
Spiritus Rector (1996b): Hannover muss zur Wiese werden! http://www.chaostage.de/ archiv/sites/1996_chc/deutsch/sr/artikel/wiese.html (letzter Zugriff am 1.2.2016).

Sprengkopf Recklinghausen (1996): Kampf der Welten! Chaostage Hannover 1995. Online unter https://vimeo.com/16711702 (letzter Zugriff am 26.1.2016).

Sutterlüty, Ferdinand (2013): Riots - moralische Eskalationen? In: WestEnd. Neue Zeitschrift für Sozialforschung 10/2, 3-23.

Thiele, Matthias (2007): Boulevard und Magazin der Normalen und der Anormalität. In: Christina Bartz / Marcus Krause (Hg.), Spektakel der Normalisierung. München: Wilhelm Fink Verlag, 103-122.

Trawny, Peter (2011): Medium und Revolution. Berlin: Matthes \& Seitz.

Trotha, Trutz von (1997): Zur Soziologie der Gewalt. In: Trutz von Trotha (Hg.), Soziologie der Gewalt. Opladen: Westdeutscher Verlag, 9-56.

Weller, Christoph (2003): Gewalt - politischer Begriff und friedenswissenschaftliche Konzepte. Eine Kritik der Gewaltfreiheit des Friedens. In: Jörg Calließ / Christoph Weller (Hg.), Friedenstheorie. Fragen - Ansätze - Möglichkeiten. Rehburg-Loccum: Evangelische Akademie, 481-508.

Westhusen, Mark M. (2005): Zonenpunkprovinz. Punk in Halle (Saale) in den 8oer Jahren. Halle (Saale): Zeit-Geschichte(n) e. V.

Žižek, Slavoj (2005): Pure Gewalt. Unkorrekte Reflexionen zu New Orleans, Frankreich und Verwandtem. In: Lettre International 71, 36-43.

Žižek, Slavoj (2011): Gewalt. Sechs abseitige Reflektionen. Hamburg: Laika.

\section{Chaos Days 1995 as a Political Spectacle. Mediality and Materiality of Urban Riots}

Twenty years ago, the so-called Chaos days shattered the city of Hannover. In the most intense civil commotions since World War II, a subcultural mélange of punks, skinheads and other factions damaged the public understanding of social order. To analyze the Chaos days in the context of an emerging riots discourse opens perspectives for material and media-related effects. Moreover it focuses on riots as disturbances and ruptures of social order perceived in the lifeworld. This is a breakdown of social sense. Because it is not immediately possible to make sense of a riot, the media began - in the case of the Chaos days - to fill the fissures, it had created. The media named, defined and pursued the genuinely modern goal of 'verstehen'. Riots are often defined and filled with sense by society and its spectacle, not by the rioter themselves. Chaos days picked up this point in a karnevalesk way: They confronted the public with an own interpretation and a distorted picture of public hysteria. 